\title{
Effects of Different Visual Feedback Forms on Eye Cursor's Stabilities
}

\author{
Xinyong Zhang ${ }^{1}$, Wenxin Feng ${ }^{1}$, and Hongbin Zha ${ }^{2}$ \\ ${ }^{1}$ School of Information, Renmin University of China, Beijing, China \\ ${ }^{2}$ Key Lab of Machine Perception, MOE, Peking University, Beijing, China \\ x.y.zhang@ruc.edu.cn, fengwenxin@gmail.com, \\ zha@cis.pku.edu.cn
}

\begin{abstract}
In this paper, we present an attention task experiment that investigated the effects of different visual feedback forms on the eye cursor's stability to find out the well-formed visual feedback. The different feedback forms were designed for dwell time, the eye cursor, and the center of the target (marked as a focus area). Our experimental findings can provide useful implications for the design of eye-controlled interfaces.
\end{abstract}

Keywords: Gaze input, visual feedback, eye cursor, stability.

\section{Introduction}

In recent years, the advancement of eye tracking technologies facilitates the applications of gaze-based interaction in different circumstances. Monitored by an eye tracker, the user's eye gaze can be used as an independent input channel to control the computer, or it also can be used as an augmented input channel in the process of human-computer interaction (HCI) to provide the user with additional assistances. However, human eyes inherently are not control but perception organs. This situation results in two typical problems of gaze input. One is that there is a lack of command activation mechanism, leading to the so-called "Midas Touch" problem of gaze input. To avoid this well-known problem, the mainstream solution requires the user to dwell on the desired target for a given time threshold.

Unfortunately, the use of dwell time as the indicator of the user's real intention can probably lead to the other problem. When the eyes are unnaturally used as control organs, they serve as both input and output channels at the same time, probably causing some inevitable conflicts. For example, the user's attention will be distracted when he/she is focusing on a target for a short while to activate the corresponding command but an accidental feedback of the system is suddenly presented on the screen. This kind of distractions will interrupt the progress of gaze-based interactions, such as the typical task of dwell-based eye pointing. Because the cursor is located at the user's gaze point on the screen and simultaneously moves with the gaze. In other 
words, the gaze input device (eye tracker) can detect the user's eye gaze in real time and the application system can synchronize the cursor with the gaze position.

Unlike the manually controlled cursor, the eye-controlled cursor is unstable and cannot be exactly fixed on the desired point. This situation is related to three factors as follows:

- Eye jitters. There is a kind of unconscious eye movements, i.e. inherent eye microtremors, even when the user is intentionally staring at a steady target. This is one of the physiological features of human eyes. This feature makes the corresponding data flow of the user's gaze points appear to be noisy.

- Performance limitations of eye tracking technology. The tracking accuracy limitation and the random errors produced by the specific algorithm of estimating gaze point also result in noises in the data flow.

- Changes in user interface. As the eyes are a kind of perception organs, the user, in general, moves his/her eyes to detect what is happening around. In graphical user interfaces, the changes, such as dynamic visual feedback, can attract the user's attention with the shift of his/her point of view.

Recently, Zhang et al. proposed a new performance model for gaze-based interactions [23] as expressed in Equation1 as follows:

$$
T=a+b \times \frac{e^{\lambda A}}{W-\mu}
$$

where $A$ and $W$ denote the movement distance of the eye cursor and the target size, respectively, both of $a$ and $b$ are regression coefficients, and the symbols $\lambda$ and $\mu$ are two empirical constants. $\lambda$ is a very small decimal (in the level of 0.0005 ), directly reflecting the feature of saccadic eye movements, i.e. the lower contribution rate of $\mathrm{A}$ to target selection time $(T) . \mu$ is a measurable parameter, denoting the average diameter of the areas where the unstable eye cursor is dwelling for target acquisitions (i.e. command activations). This parameter probably can vary in different situations, e.g. different gaze input devices. The fraction term is defined as the index of difficulty $\left(I D_{\text {eye }}\right)$ for dwell-based eye pointing like the well-known logarithm term in Fitts' law for hand pointing. Pointing task is the dominant task type in different graphical user interfaces. As Zhang et al.'s experiments revealed, the correlation between $I D_{\text {eye }}$ and $\mathrm{T}$ was strong enough $\left(R^{2}>0.9\right)$. One of the implications of the model is that the eye cursor's stability can significantly affect the user's capability of dwell-based eye pointing. According to the aforementioned factors causing the eye cursor to be unstable, besides designing feasible underlying algorithms to alleviate the effect of the signal noises $[15,22]$, it is also very necessary to carefully take account of the design of visual feedback because in graphical user interfaces (GUI), visual feedback is a useful form to inform the user whether or not the system is under control as expected, regardless of the possibility that visual feedback can disturb, frustrate and even defeat the process of gaze input.

In this paper, therefore, we present an attention task experiment that investigated the effects of different visual feedback forms on the eye cursor's stability. In our experiment, the visual feedback was designed for the elapse of dwell time, the visibility of the eye cursor, and the center of the target (marked as a focus area or not). 
Our purpose is to find out the well-formed visual feedback that can benefit gaze input without significantly distracting the user so as to avoid the deterioration of the stability of the eye cursor. Our experimental findings can provide useful implications for the design of eye-controlled interfaces.

\section{Related Work}

The stability of the eye cursor is a significant factor that can affect the human performance in gaze-based interactions [22]. In order to improve the eye cursor's stability, Zhang et al. proposed several simple but effective algorithms to constrain the noises of eye gaze input signals. Unlike Kumar et al.'s efforts of filtering the noises using different algorithms [15], such as a Kalman filter with saccade detection, the key of Zhang et al.'s solutions was to make the signal noise "recessive", without the need of a specific algorithm to detect saccadic eye movements. Therefore, there was no predetermined time window required for the detection of saccades or the other types of eye movements. In this situation, the gaze input signals almost can be synchronous with the user's eye movements, without damping the speed of eye movements.

Zhang et al.'s work as well as that of Kumar et al. processed the raw data of gaze input using underlying algorithms. It was coincident with the observation of Majaranta et al. that the majority of the research on eye tracking applications did not concentrate on the UI design issues but on the basic technical aspects in the past of the last decade $[17,18]$. With the advancement of eye tracking techniques, there had been a number of applications of gaze input, such as the traditional application of text entry (i.e. eye typing) [21, 17] and the novel applications of reading assistant $[9,19]$, coordination of multiple applications or systems [4, 1], game entertainments [20], mobile environment [2], virtual reality [11, 8] and even personal privacy and information security [13]. Therefore, it is necessary to carefully take account of the design issues in gaze input user interfaces, especially the use of visual feedback because an improper visual feedback can probably affect the user's gaze behavior so as to frustrate the process of gaze input.

The necessity of visual feedback for HCI is well-known. Visual feedback is a meaningful cue for the user to know the current state of interactive systems and infer whether they are responding to the user's action as the anticipation or not. In gazebased interaction systems, visual feedback is also often used for different purposes [17]. Istance et al. pointed out that the user could be explicitly aware of which target on the screen was "captured" by his/her gaze, avoiding the accidental activation of undesired commands, when given a clear feedback [10]. Unlike hands, there is no explicit command activation mechanism for human eyes, resulting in the well-known "Midas Touch" problem of gaze based interactions [12]. Requiring the user to continuously stare at the desired target for a predetermined amount of time (e.g. 900 ms, called dwell time), rather than to consciously blink during the stare, is still the dominant means to activate command and avoid the "Midas Touch" problem in the situation of hand free gaze input. In order to indicate the progress of dwell time, Lankford designed a red shrinking rectangle to highlight the key, on which the user fixated in the on-screen keyboard of the ERCIA eye typing system [16]. At the end of 
the dynamic shrinking, the corresponding character was entered just like that the key was hit. This form of visual feedback provided a continuous feedback when the user is waiting for the activation of the key. Regardless of the tedious prolonged dwell time, Lankford also employed different forms of visual feedback at different stages during the progress of dwell time for the user to perform the equivalent actions of click, drag and drop, double click.

Majaranta et al. refined the shrinking form of visual feedback in their eye typing system [18]. They directly shrank the centered caption letter of the key instead of an additional rectangle. Their evaluation experiment indicated that shrinking the letter could help the user steadily focus on the center of the key until successfully "hitting" the key. That is to say, this visual feedback form could partly prevent the common phenomenon of eye typing that the user often glances at the next key before entering the current letter. As the improved gaze behavior they reported, the occurrences of refocusing to repair the unsuccessful keystroke can be decreased, leading to the increase of typing speed. Note that, however, the drift of the user's gaze from the current key to the next is mainly due to his/her clear consciousness about the letter sequence of the word that the user is inputting. This subconsciousness gives the user an impulse to move the eyes to the next "target" before satisfying the criterion of dwell time. It appears that the user generates a trained reflex from the visual feedback to counteract the subconsciousness. In other words, the events of refocusing can be consciously reduced by the user. Majaranta et al.'s experiment revealed this fact. It did not yet sufficiently expose the impacts of only visual feedback itself on the user's gaze behavior in the situation without the impulse of subconsciousness.

Using a traditional progress bar in the GazeTalk eye typing system [6, 7], Hansen et al. also provided a dynamic visual feedback to inform the user the remaining time before the activation of the key. In this system, they did not employ a standard QWERTY layout for the on-screen keyboard, thus the keys presented in the dynamical layout were big enough to compensate for the low accuracy of gaze input. They were aware of that the highlighted progress bar likely could lead the user's eyes to follow the move of the bar, but they did not provide a detailed experimental analysis like the work of Majaranta et al. to support this point.

As introduced in the beginning of this section, Zhang et al. addressed the issue of unstable eye cursor from the perspective of signal processing [22]. They carried out an attention task experiment to evaluate the effectiveness of their methods. In their previous experiment, they also took account of the factor of visual feedback. They designed a semi-transparent red "bubble" covering on the target as the medium of visual feedback. The bubble had two modes to indicate the elapse of trial time in the attention task. One mode was that the bubble expands from the center of the target at the beginning of trials to the edge of the target at the end, the other was that the bubble, fully filling the target at the beginning, contrarily shrinks to the center during each trial. Since Zhang et al. just needed to confirm that the visual feedback they used in the other pointing task experiment would not bias the effectiveness of the gaze signal processing algorithms; their attention task experiment did not carefully considered the effects of different visual feedback forms on the cursor's stability. Nevertheless, their previous work provided some useful insights for our current work, 
such as the measures from both temporal and spatial perspectives to describe the cursor's stability.

\section{Attention Task Experiment}

The eye cursor's stability is directly related to the user's gaze behaviour (i.e. the data flow of gaze points), while the gaze behaviour can be affected by the visual communication from the computer to the user. Therefore, it is necessary to reveal the specific effects of visual feedback on eye cursor's stability. We carried out a similar attention task experiment to that in Zhang et al.'s previous work [22], but we deliberately considered the forms of visual feedback for different aspects of gaze input interfaces, including the elapse of invisible dwell time, the visibility of eye cursor and the use of focus area in the target.

\subsection{Apparatus and Participants}

The software and hardware configurations of the experiment were similar to those in Zhang et al.'s work [22]. We employed a head mounted eye tracker, EyeLink II, as the gaze input device. It worked in pupil only mode at the sampling rate of $250 \mathrm{~Hz}$. The tasks were presented on a 19-inch CRT display at $1024 \times 768$ resolution. Sixteen ablebodied participants ( 8 females and 8 males), with the average age of 24 , successfully completed this experiment. They had normal or corrected-to-normal vision.

\subsection{Task and Procedure}

Before the beginning of the experiment, the experimenter seated the subject in front of the display. There was a table, of which the width is about $70 \mathrm{~cm}$, between the subject and the screen to maintain a fixed distance. The subject was permitted to adjust the chair to suitable position and height, and she/he also could place the hands on the table to support her/his head. After giving a brief introduction about the task, the experimenter launched and calibrated the gaze input device, and then formally started the experiment. There was no training since the task was intuitive and simple for the subject to perform.

As Figure 1a shows, at the very onset of the experiment, a trial-start button, without being enabled, was displayed at the center of the screen. The experimenter pressed the ' $\mathrm{S}$ ' key to enable the trial-start button, and then the button could be moved among the nine predefined positions in turn. The trial-start button was rendered as a 24-pixel-diameter round, but its effective diameter was 100 pixels. Once the subject focused on the trial-start button for a very short time $(450 \mathrm{~ms})$, it disappeared with the target displayed at the same position and the trial started. The subject was explicitly asked to fixate on the center of the target for 2.5 seconds until the end of the trial. At the same time the subject was also instructed not to chase the visible eye cursor. When the trial ended, the target disappeared and the trial-start button was redisplayed at the next position to repeat the process above.

During the trial, the experimenter was able to suspend the experiment and perform a recalibration if the calibration deteriorated. When resuming the experiment, the 


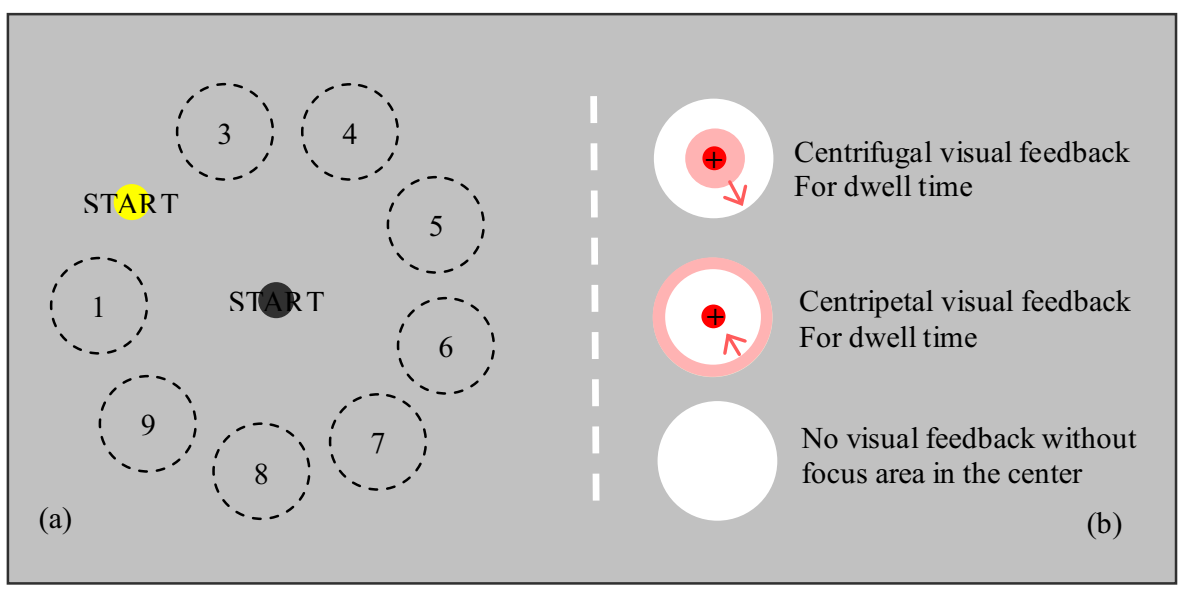

Fig. 1. (a) The experiment interface, the dashed circles just illustrate the positions of the target. (b) The target with different visual feedback modes

experimenter was also able to reset the position of the trial-start button. This provided a chance to undo and redo the trials when affected by the deterioration of calibration.

\subsection{Design}

This experiment investigated the effects of the following factors at different levels:

- Visual feedback mode of dwell time (VF): As Figure 1b depicts, one mode was that the semi-transparent red color spread from the center of target $(V F 1)$, while the other spread from the edge $(V F 2)$. Both of the modes had the same end state that the target was fully covered. The mode of no feedback was treated as a baseline for comparisons ( $V F O)$.

- Target diameter (TD): The size of the target was at four levels of 36, 46, 60, and 78 pixels.

- Focus area in target (FA): The center of the target was marked using a red small round to help the user focus on the target.

- Eye cursor's style (CS): Three cursor styles, including cross cursor, arrow cursor and invisible cursor, were took into account.

Consequently, the experiment was a $(3 \times 4 \times 2) \times 3$ repeated measures within-subject design. The first three factors resulted in 24 combinations. These combinations presented in a random order during the experiment. For each combination, there were 9 trials resulted from three repetitions for each of the eye cursor conditions. Those trials were performed at the 9 positions as indicated in Figure 1a, respectively, but the order of the visual feedback modes was counterbalanced among the positions. All the 24 combinations, totally including 216 trials, composed a block. The subject needed to finish 4 blocks of trials within one session of about an hour. 


\subsection{Results}

In order to analyze the stability of the eye cursor, we used three dependent variables as Zhang et al. previously used [22,23].
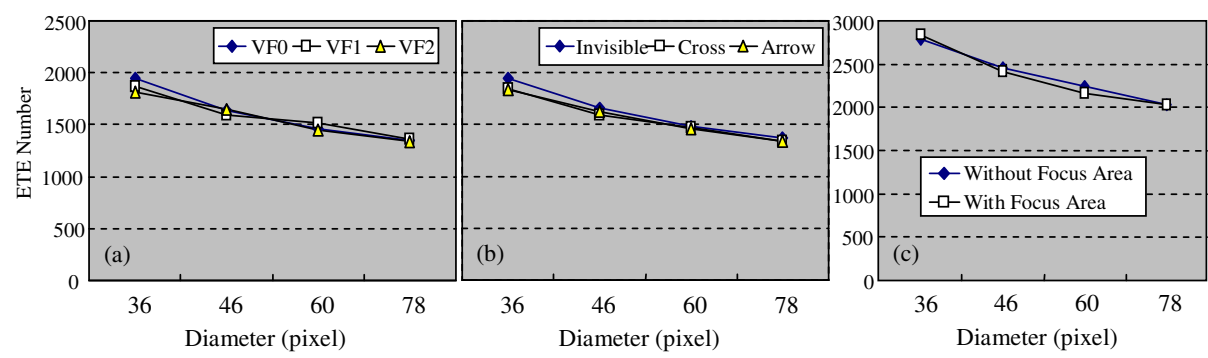

Fig. 2. ETE number by diameter under different (a) visual feedback conditions of dwell time, (b) eye cursor styles, and (c) focus area conditions

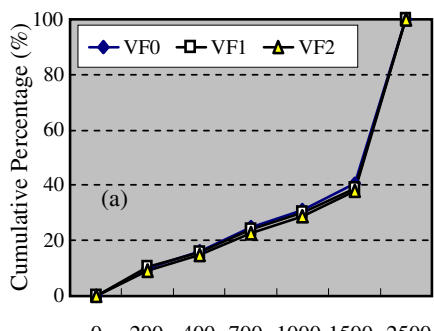

$0 \quad 200 \quad 400700 \quad 10001500 \quad 2500$

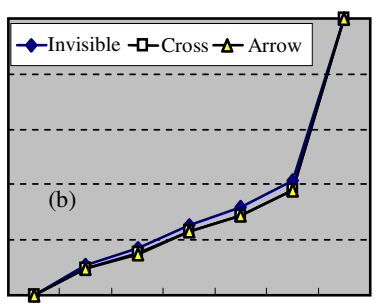

$0 \quad 200400 \quad 700100015002500$

Dwell Time (ms)

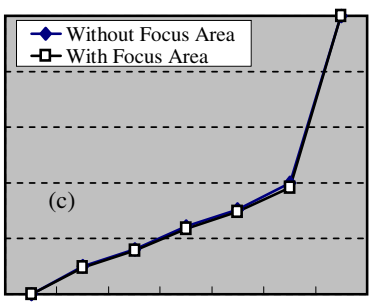

$\begin{array}{llllll}0 & 200 & 400 & 700 & 100015002500\end{array}$

Fig. 3. Cumulative percentage of dwell times under different visual feedback conditions

Eye Cursor's Entering Target Event (ETE). The unstable eye cursor can probably repeatedly enter and leave the effective area of the target. We recorded this kind of event when it happened. We found that there was no significant main effect for the three factors of visual feedback on ETE but only a significant interaction effect VF $\times$ TD $\left(\mathrm{F}_{6,90}=2.43, \mathrm{p}<.05\right)$. As Figure 2 shows, the different forms of visual feedback did not lead to obvious differences.

Frequency Distribution of Eye Cursor's Dwell Time. Corresponding to ETE, we also recorded the durations when the cursor was inside of the target and count the frequencies of the durations in different ranges as Figure 3 illustrates. Therefore, this is a multivariate measures. It was revealed that there was no significant main effect for all the factors but target size (TD) on this multivariate measure. Univariate test for each dimension also did not observe significant main effect except for the statistically significant effect of the factor $\mathrm{CS}\left(\mathrm{F}_{2,30}=3.50, \mathrm{p}=.043\right)$ in the first range. Figure 3 plots the total cumulative percentage of dwell times under different visual feedback conditions, without showing obvious differences. 


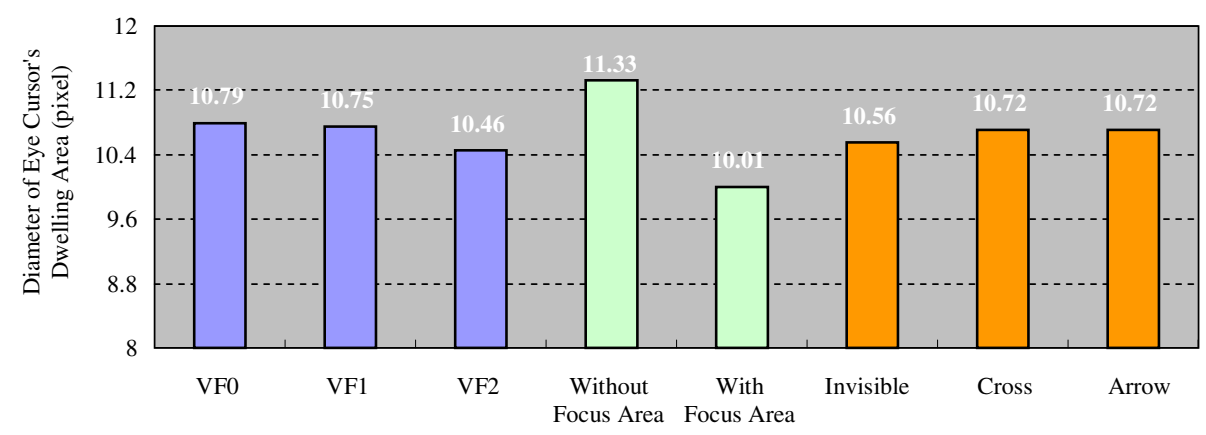

Different Visual Feedback Forms

Fig. 4. Average diameter of dwelling areas under different visual feedback conditions

Diameter of Eye Cursor's Dwelling Area. For each trial, when the eye cursor entered the target, its positions were sampled at the rate of 25 points per second. Totally, we collected about five hundred thousand points. According to the sampled points, the average diameter of the cursor's dwelling area in each trial was calculated. We found that there was significant effect for the factor $\mathrm{VF}\left(\mathrm{F}_{2,30}=5.87, \mathrm{p}<.01\right)$ as well as FA $\left(\mathrm{F}_{1,15}=85.97, \mathrm{p}<.001\right)$ on this measure. As Figure 4 shows, it was useful for stabilizing the eye cursor to mark the center of the target as a focus area or to use a centripetal visual feedback, while it did not deteriorate the stability to present a visible cursor because the factor CS had no significant main effect $\left(\mathrm{F}_{2,30}=0.57, \mathrm{p}=.571\right)$.

\section{Discussion}

Although a cursor, following the user's eye gaze, was rendered in some gaze-based interactive systems [14], some researchers [12,5] argued that a visible cursor was unsuitable for gaze-based interactions because they believed that the user's attention would be distracted especially when the cursor was not accurately located at the user's real gaze point due to errors. However, our experimental results indicated that a visible cursor did not distract the user attention even if there was no focus area in the target. In other words, the user was able to avoid chasing the visible cursor when given an explicit instruction. Furthermore, another study indicated that the human performance could be improved when a visible eye cursor was used [3]. Therefore, presenting the cursor will not damage the usability of gaze-based interactive systems.

Kumar et al. investigated the use of focus points $[14,15]$, but unfortunately they did not observed a significant effect. Our experiment indicated that using a relatively big area to mark the center of the target, in stead of a grid of small focus points covered on the whole interface, could make the user's gaze points more concentrated, i.e. could stabilize the eye cursor.

Compared with the similar task in Zhang et al.'s experiment [22], of which the trial time was 7 seconds, the time we currently used was shorter. Note that we human beings blink about 12 times every minute in general [24]. The experiment's trial time, 2.5 seconds, did not exceed the average interval of natural blinks. This could largely prevent the experimental results from being biased by the subjects' blinks. 


\section{Conclusions}

Visual sense is the main channel that we human beings perceive the world. When the visual channel is unnaturally used for input (control) in HCI, some potential problems probably could be raised. In this paper, we present an attention task experiment to investigate the effects of different visual feedback forms. Based on a large number of data, our experimental findings clarify that a visible eye cursor will not degrade the usability of gaze-based interactions. Our experiment also indicates that a proper dynamic visual feedback for dwell time and a static focus area in the target can benefit gaze input.

Acknowledgments. This work was supported by the National Natural Science Foundation of China (Grant No. 61070144), and also was partly supported by the Fundamental Research Funds for the Central Universities and the Research Funds of Renmin University of China (Grant No. 10XN1029) for the project of Research on Financial Web Data Mining and Knowledge Management.

\section{References}

1. Dickie, C., Hart, J., Vertegaal, R., Eiser, A.: LookPoint: an evaluation of eye input for hands-free switching of input devices between multiple computers. In: OZCHI 2006, Sydney, Australia, pp. 119-126. ACM Press, New York (2006)

2. Drewes, H., Luca, A.D., Schmidt, A.: Eye-gaze interaction for mobile phones. In: Mobility Conference 2007, Singapore, pp. 364-371. ACM Press, New York (2007)

3. Feng, C., Shen, M., Chen, S., Su, H.: Effects of target border and eye cursor on human performance in eye-based interactions (in Chinese). Chinese Journal of Applied Psychology 10(1), 9-12 (2004)

4. Fono, D., Vertegaal, R.: Eyewindows: Evaluation of eye-controlled zooming windows for focus selection. In: CHI 2005, Portland, Oregon, USA, pp. 151-160. ACM Press, New York (2005)

5. Hatfield, F., Jenkins, E.A., Jennings, M.W., Calhoun, G.: Principles and guidelines for the design of eye/voice interaction dialogs. In: The IEEE 3rd Symposium on Human Interaction with Complex Systems (HICS 1996), Dayton, USA, pp. 10-19. IEEE Press, Los Alamitos (1996)

6. Hansen, J.P., Johansen, A.S., Hansen, D.W., Itoh, K., Mashino, S.: Command without a click: dwell time typing by mouse and gaze selections. In: INTERACT 2003, Zurich, Switzerland, pp. 121-128. IOS Press, Amsterdam (2003)

7. Hansen, J.P., Tørning, K., Johansen, A.S., Itoh, K., Aoki, H.: Gaze typing compared with input by head and hand. In: ETRA 2004, San Antonio, Texas, USA, pp. 131-138. ACM Press, New York (2004)

8. Haffegee, A., Barrow, R.: Eye tracking and gaze based interaction within immersive virtual environments. In: Allen, G., Nabrzyski, J., Seidel, E., van Albada, G.D., Dongarra, J., Sloot, P.M.A. (eds.) ICCS 2009. LNCS, vol. 5545, pp. 729-736. Springer, Heidelberg (2009)

9. Hyrskykari, A., Majaranta, P., Aaltonen, A., Räihä, K.-J.: Design issues of idict: a gazeassisted translation aid. In: ETRA 2000, Palm Beach Gardens, Florida, USA, pp. 9-14. ACM Press, New York (2000) 
10. Istance, H.O., Spinner, C., Howarth, P.A.: Providing motor impaired users with access to standard graphical user interface (GUI) software via eye-based interaction. In: The 1st European Conference on Disability, Virtual Reality and Associated Technologies (ECDVRAT 1996), Maidenhead, UK, pp. 109-116 (1996)

11. Istance, H., Bates, R.: Towards eye based virtual environment interaction for users with high-level motor disabilities. In: The 5th Intl. Conf. on Disability, Virtual Reality and Associated Technologies (ICDVRAT), Oxford, UK, pp. 275-282 (2004)

12. Jacob, R.J.K.: Eye movement-based human-computer interaction techniques: Toward noncommand interfaces. In: Advances in Human-Computer Interaction, vol. 4, pp. 151-190. Ablex Publishing, Greenwich (1993)

13. Kumar, M., Garfinkel, T., Boneh, D., Winograd, T.: Reducing shoulder-surfing by using gaze-based password entry. In: The 3rd Symposium on Usable Privacy and Security, Pittsburgh, PA, USA, pp. 13-19. ACM Press, New York (2007)

14. Kumar, M., Paepcke, A., Winograd, T.: Eyepoint: Practical pointing and selection using gaze and keyboard. In: CHI 2007, San Jose, California, USA, pp. 421-430. ACM Press, New York (2007)

15. Kumar, M., Klingner, J., Puranik, R., Winograd, T., Paepcke, A.: Improving the accuracy of gaze input for interaction. In: ETRA 2008, pp. 65-68. ACM Press, Savannah (2008)

16. Lankford, C.: Effective eye-gaze input into windows. In: ETRA 2000, Palm Beach Gardens, Florida, USA, pp. 23-27. ACM Press, New York (2000)

17. Majaranta, P., Räihä, K.-J.: Twenty years of eye typing: systems and design issues. In: ETRA 2002, New Orleans, Louisiana, USA, pp. 15-22. ACM Press, New York (2002)

18. Majaranta, P., MacKenzie, I.S., Aula, A., Räihä, K.-J.: Effects of feedback and dwell time on eye typing speed and accuracy. Journal of Universal Access in the Information Society 5(2), 199-208 (2006)

19. Sibert, J.L., Gokturk, M., Lavine, R.A.: The reading assistant: eye gaze triggered auditory prompting for reading remediation. In: UIST 2000, San Diego, CA, USA, pp. 101-107. ACM Press, New York (2000)

20. Smith, J.D., Graham, T.C.: Use of eye movements for video game control. In: ACE 2006, article No. 20, Hollywood, CA, USA, ACM Press, New York (2006)

21. Wang, J., Zhai, S., Su, H.: Chinese input with keyboard and eye tracking: an anatomical study. In: CHI 2001, Seattle, WA, USA, pp. 349-356. ACM Press, New York (2001)

22. Zhang, X., Ren, X., Zha, H.: Improving eye cursor's stability for eye pointing tasks. In: CHI 2008, Florence, Italy, pp. 525-534. ACM Press, New York (2008)

23. Zhang, X., Ren, X., Zha, H.: Modeling dwell-based Eye pointing target acquisition. In: CHI 2010, Atlanta, GA, USA, pp. 2083-2092. ACM Press, New York (2010)

24. The Human Body - How Often Does The Human Eye Blink? Science Fact Finder. In: Engelbert, P. (ed.) UXL-Gale, 1998. eNotes.com. (2006), http: / / www. enotes.com/science-fact-finder/human-body/ how-often-does-human-eye-blink (February 24, 2011) 